\title{
Sigmoid colon cancer with an incidental diagnosis of Leriche syndrome successfully treated without inferior epigastric artery injury
}

\author{
Naoto Mizumura*, Masayasu Kawasaki, Satoshi Okumura, Sho Toyoda, AtsuoImagawa, and Masao Ogawa \\ Department of Surgery, Bellland General Hospital, Japan
}

\begin{abstract}
Leriche syndrome involves atherosclerotic occlusion of the infrarenal aorta and bilateral common iliac arteries, and the inferior epigastric artery is an important route for lower-extremity perfusion. A 65-year-old Japanese man with sigmoid colon cancer was scheduled to undergo laparoscopic colectomy, and three-dimensional computed tomography angiography was performed to detect the feeding artery of the tumor. The angiography incidentally showed occlusion of the infrarenal aorta and common iliac arteries with the following collateral pathways: the internal thoracic artery supplied the external iliac artery via the inferior epigastric artery and the intercostal arteries supplied the external iliac artery via the superficial circumflex iliac artery. Therefore, he was diagnosed with Leriche syndrome. We performed open colectomy with midline incision and colostomy at the midline, without drain placement to prevent inferior epigastric artery injury. In patients with Leriche syndrome, this approach might be a safe option to avoid lower extremity ischemia.
\end{abstract}

\begin{abstract}
Abbreviations: ITA: internal thoracic artery; IEA: inferior epigastric artery; CT: computed tomography; 3D: three-dimensional

\section{Background}

Leriche syndrome, also referred to as aortoiliac occlusive disease, involves atherosclerotic occlusion of the infrarenal aorta and bilateral common iliac arteries. In patients with Leriche syndrome, an internal thoracic artery (ITA) to inferior epigastric artery (IEA) collateral pathway is present, which is an important route providing lowerextremity perfusion [1]. In cardiovascular surgery, the ITA is often used as a coronary artery graft, and injury to the ITA-IEA route is known to cause lower extremity ischemia [1]. However, in gastroenterological surgery, the consequences of injury to the ITA-IEA route are not well known. The IEA can be injured during invasive procedures, such as surgery, paracentesis, and percutaneous drain placement [2]. Here we present a case of sigmoid colon cancer with an incidental diagnosis of Leriche syndrome, which was successfully treated without IEA injury.
\end{abstract}

\section{Case presentation}

A 65-year-old Japanese man presented to our hospital because of hematochezia. He was bedridden for 13 years because of cerebral infarction, with total aphasia and right hemiplegia. Laboratory results showed normal hemoglobin levels, renal function, liver function, carcinoembryonic antigen levels, and carbohydrate antigen 19-9 levels. Colonoscopy revealed a type 2 tumor localized in the sigmoid colon, and pathological examination identified the tumor as a moderately differentiated tubular adenocarcinoma. Contrast-enhanced computed tomography $(\mathrm{CT})$ revealed a mass measuring approximately $50 \times 40$ $\mathrm{mm}$ in the sigmoid colon, without enlargement of the lymph nodes or metastases to the liver and lungs. According to the UICC classification, the tumor was classified as T4aN0M0, stage II B [3].

Laparoscopic colectomy was planned. We performed three- dimensional (3D) CT angiography to detect the feeding artery of the tumor, and it incidentally showed occlusion of the infrarenal aorta and common iliac arteries with the following collateral pathways: the ITA supplied the external iliac artery via the IEA and the intercostal arteries supplied the external iliac artery via the superficial circumflex iliac artery (Figure 1). The origin of the inferior mesenteric artery was occluded. The middle colic artery supplied blood to the inferior mesenteric artery territory through the marginal artery of Drummond (Figure 2). Therefore, he was diagnosed with Leriche syndrome (TASC type D iliac lesion) [4]. His femoral pulse was diminished, and the ankle brachial index was reduced (right, 0.44; left, 0.47). Considering his general condition, and the absence of rest pain, necrosis, or gangrene, surgery was not indicated for his Leriche syndrome.

We planned Hartmann's operation, and the stoma site was marked preoperatively at the epigastric region. A partial colectomy with paracolic lymph node dissection was performed after midline incision, and transverse loop colostomy was performed at the midline wound (Figure 3). A drainage tube was not placed. The pathological result of the paracolic lymph nodes was negative, and we finally classified the tumor as T4aN0M0, stage II B. The ankle brachial index did not deteriorate after the procedure, and he was discharged without any

Correspondence to: Dr. Naoto Mizumura, Department of Surgery, Bellland General Hospital, 500-3 Higashiyama, Naka-ku, Sakai-city, Osaka 5998247, Japan, Tel: +81 (0)72 234 2001, Fax: +81 (0)72 234 2003; E-mail: n_mizumura@seichokai.or.jp

Key words: colon cancer, inferior epigastric artery, Leriche syndrome, lower extremity ischemia

Received: October 06, 2015; Accepted: October 21, 2015; Published: October 23,2015 


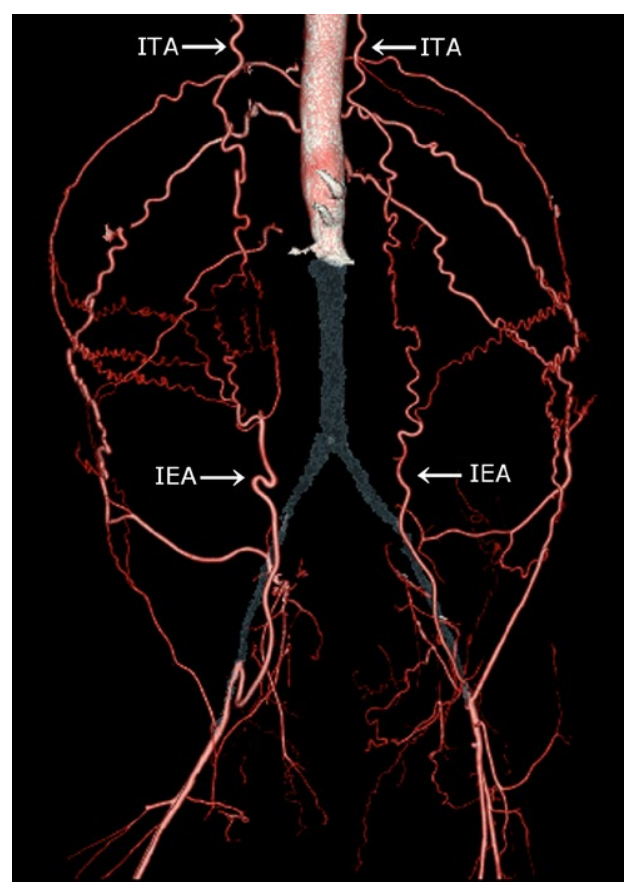

Figure 1. Occlusion of the infrarenal aorta and bilateral common iliac arteries.

The grey territory shows occlusion of the infrarenal aorta and bilateral common iliac arteries. The internal thoracic artery supplies the external iliac artery via the inferior epigastric artery.

ITA, internal thoracic artery; IEA, inferior epigastric artery

complications on postoperative day 21 . He has not shown any signs of recurrence at the 30-month follow-up.

\section{Discussion}

We presented a case of sigmoid colon cancer with an incidental diagnosis of Leriche syndrome, which was successfully treated without IEA injury. This case highlights that 3D CT angiography is useful for the diagnosis of Leriche syndrome and for the assessment of vascular anatomy before colorectal surgery in such cases. Leriche syndrome classically involves a history of the following triad of symptoms: claudication, impotence, and decreased lower limb pulse. The hallmark of Leriche syndrome is the symptom of claudication in most cases. Our patient had no subjective symptoms because of his bedridden status with total aphasia. On CT for preoperative evaluation of colon cancer, the clinicians and interpreting radiologist overlooked Leriche syndrome owing to the absence of clinical information. However, 3D CT angiography incidentally revealed Leriche syndrome. A previous study reported that CT angiography has an overall sensitivity and specificity of $96 \%$ and $97 \%$, respectively, for Leriche syndrome [5]. The possibility of Leriche syndrome should be considered in bedridden patients.

The inferior mesenteric artery and its anastomoses have several variations in their origins and branches. Therefore, preoperative visualization of $3 \mathrm{D}$ reconstructions of the mesenteric vessels can help prevent complications [6]. Commonly, regional lymph node dissection is performed up to the root of the inferior mesenteric artery in patients with advanced sigmoid colon cancer. In the present case, the origin of the inferior mesenteric artery was occluded and the middle colic artery provided blood supply to the sigmoid colon cancer through the marginal artery of Drummond. The appropriate regional lymph node dissection in such cases is not clear. Additionally, it is possible that only the superior rectal artery provided blood supply to the rectum because the middle and inferior rectal arteries had poor blood flow owing to the internal iliac artery occlusion. Our patient had no rectal ischemia owing to paracolic lymph node dissection with preservation of the superior rectal artery and left colic artery (Figure 2).

The present case also highlights that attention should be paid to IEA injury in patients with Leriche syndrome. Leriche syndrome involves an ITA to IEA collateral pathway that provides lowerextremity perfusion [1]. In abdominal surgery, the incision should be carefully made because the epigastric artery lies behind the rectus abdominis muscle. All incisions (transverse, subcostal, and pararectus incisions), except for midline incisions, can result in IEA injury [7]. Laparoscopic surgery has become increasingly popular in recent years, and it has been reported that trocar injury to vessels of the abdominal wall occurs in $0.2-2 \%$ of laparoscopic procedures [7]. IEA injury has been reported to be the one of the most common complications, and it cannot be effectively located with laparoscopic transillumination [8]. If

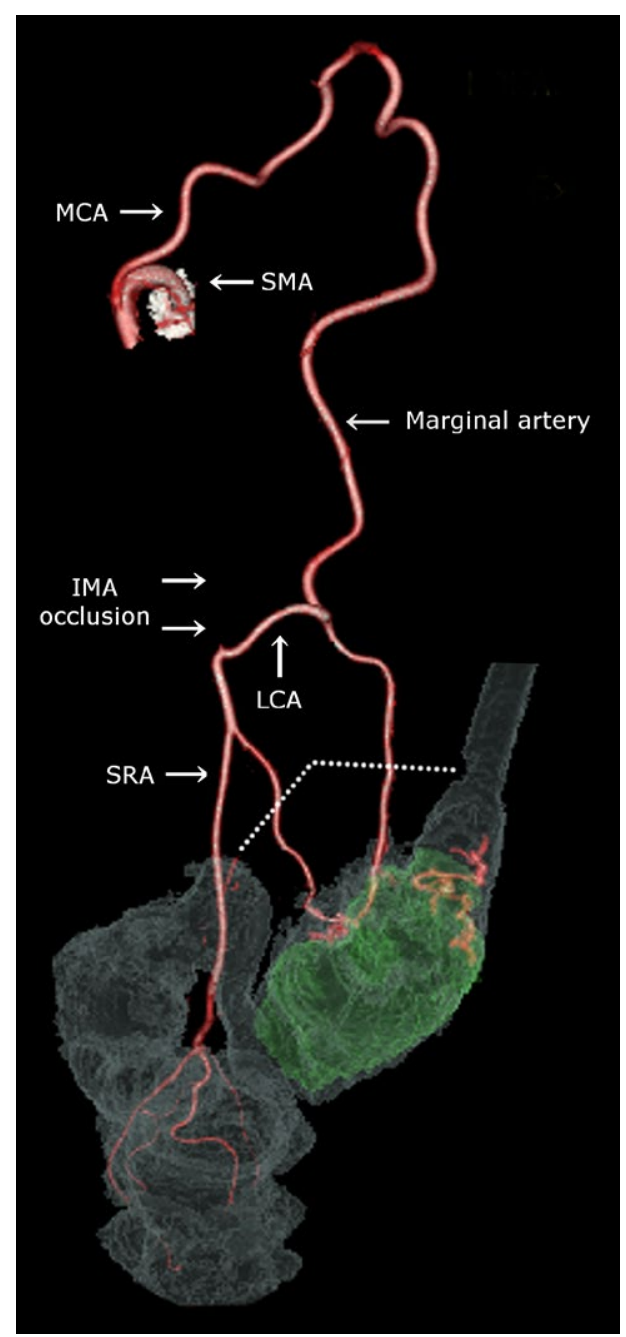

Figure 2. The feeding artery of the sigmoid colon cancer.

The green territory shows the sigmoid colon cancer. The origin of the inferior mesenteric artery is occluded. The middle colic artery supplies blood to the inferior mesenteric artery territory through the marginal artery. The dotted line indicates the resection line.

SMA, superior mesenteric artery; MCA, middle colic artery; LCA, left colic artery; SRA, superior rectal artery; IMA, inferior mesenteric artery 


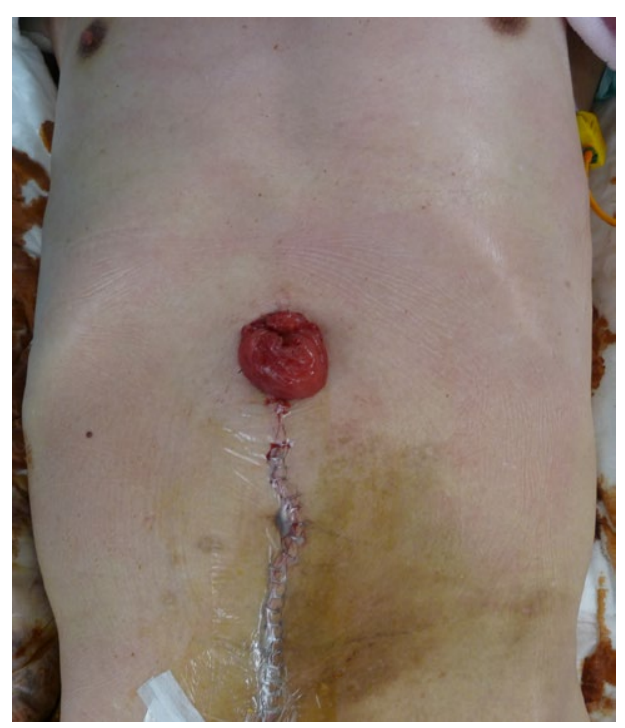

Figure 3. Colostomy at the epigastric region.

laparoscopic surgery is required in patients with Leriche syndrome, the IEA should be ultrasonographically identified before trocar insertion. Similarly, in patients with Leriche syndrome, ostomy surgery should be carefully performed to avoid IEA injury. In patients with a poor general condition, colostomy is often performed to prevent anastomotic leakage and fecal incontinence, and the stoma generally passes through the rectus abdominis muscle. A previous study reported life-threatening hemorrhage from IEA injury after stoma construction [9]. Therefore, in such cases, open surgery with midline incision, colostomy at the midline, and no drain placement can help avoid IEA injury.

\section{Conclusions}

We reported a case of sigmoid colon cancer with an incidental diagnosis of Leriche syndrome, which was successfully treated without IEA injury. Clinicians should be aware that Leriche syndrome might remain unrecognized in bedridden patients. In the present case, 3D CT angiography was useful for the diagnosis of Leriche syndrome and for the assessment of the vascular anatomy before colorectal surgery. We performed open surgery with midline incision and colostomy at the midline, without drain placement to prevent IEA injury. In patients with Leriche syndrome, this approach might be a safe option to avoid lower extremity ischemia.

\section{Authors' contributions}

NM and MK performed the operation and drafted the manuscript. SO, ST, AI, and MO revised the manuscript. All authors conceived of this case report. All authors read and approved the final manuscript.

\section{Acknowledgements}

The authors would like to thank Akiko Yamana(Certified Nurse in Wound, Ostomy and Continence Nursing) for stoma site preparation and stoma care.

\section{Funding}

No funding was received.

\section{Conflicts of interest}

None declared.

\section{References}

1. Yurdakul M, Tola M, Ozdemir E, Bayazit M, Cumhur T (2006) Internal thoracic arteryinferior epigastric artery as a collateral pathway in aortoiliac occlusive disease. $J$ Vasc Surg 43: 707-713. [Crossref]

2. Sobkin PR, Bloom AI, Wilson MW, LaBerge JM, Hastings GS, et al. (2008) Massive abdominal wall hemorrhage from injury to the inferior epigastric artery: a retrospective review. J Vasc Interv Radiol 19: 327-332. [Crossref]

3. Sobin LH, Gospodarowicz MK, Wittekind C. 2009. TNM Classification of Malignant Tumours vol. 7. Oxford: Willey-Blackwell.

4. Norgren L, Hiatt WR, Dormandy JA, Nehler MR, Harris KA, et al. 2007. Inter-society consensus for the management of peripheral arterial disease (TASC II). Eur J Vasc Endovasc Surg 45: S1-75. [Crossref]

5. Heijenbrok-Kal MH, Kock MC, Hunink MG (2007) Lower extremity arterial disease multidetector CT angiography meta-analysis. Radiology 245: 433-439. [Crossref]

6. Mari FS, Nigri G, Pancaldi A, De Cecco CN, Gasparrini M, et al. (2013) Role of CT angiography with three-dimensional reconstruction of mesenteric vessels in laparoscopic colorectal resections: a randomized controlled trial. Surg Endosc 27: 2058-2067. [Crossref]

7. Saber AA, Meslemani AM, Davis R, Pimentel R (2004) Safety zones for anterior abdominal wall entry during laparoscopy: a CT scan mapping of epigastric vessels. Ann Surg 239: 182-185. [Crossref]

8. Quint EH, Wang FL, Hurd WW (1996) Laparoscopic transillumination for the location of anterior abdominal wall blood vessels. J Laparoendosc Surg 6: 167-169. [Crossref]

9. Yamagami T, Yoshimatsu R, Tanaka O, Miura H, Ito T, et al. (2011) Transcatheter arterial embolization for hemorrhage from the inferior epigastric artery injured after stoma construction. Jpn J Radiol 29: 144-147. [Crossref]

Copyright: (2015 Mizumura N. This is an open-access article distributed under the terms of the Creative Commons Attribution License, which permits unrestricted use, distribution, and reproduction in any medium, provided the original author and source are credited. 